\title{
Sharp and general estimates for the Bellman function of three integral variables related to the dyadic maximal operator
}

\author{
Anastasios D. Delis, Eleftherios N. Nikolidakis
}

\begin{abstract}
We compute the Bellman function of three integral variables associated to the dyadic maximal operator on a subset of its domain. Additionally, we provide an upper bound for the whole domain of its definition.
\end{abstract}

\section{Introduction}

It is well known that the dyadic maximal operator on $\mathbb{R}^{n}$ is a useful tool in analysis and is defined by

$$
\mathcal{M}_{d} \phi(x)=\sup \left\{\frac{1}{|Q|} \int_{Q}|\phi(y)| \mathrm{d} y: x \in Q, Q \subseteq \mathbb{R}^{n} \text { is a dyadic cube }\right\},
$$

for every $\phi \in L_{\text {loc }}^{1}\left(\mathbb{R}^{n}\right)$, where the dyadic cubes are those formed by the grids $2^{-N} \mathbb{Z}^{n}$, for $N=0,1,2, \ldots$. It is also well known that it satisfies the following weak type $(1,1)$ inequality

$$
\left|\left\{x \in \mathbb{R}^{n}: \mathcal{M}_{d} \phi(x)>\lambda\right\}\right| \leq \frac{1}{\lambda} \int_{\left\{\mathcal{M}_{d} \phi>\lambda\right\}}|\phi(y)| \mathrm{d} y,
$$

for every $\phi \in L^{1}\left(\mathbb{R}^{n}\right)$ and every $\lambda>0$, and which is easily proved to be best possible. Further refinements of (1.2) can be seen in [10] and [11].

Then by using (1.2) and the well known Doob's method it is not difficult to prove that the following $L^{p}$ inequality is also true

$$
\left\|\mathcal{M}_{d} \phi\right\|_{p} \leq \frac{p}{p-1}\|\phi\|_{p}
$$

for every $p>1$ and $\phi \in L^{p}\left(\mathbb{R}^{n}\right)$. Inequality (1.3) turns out to be best possible and its sharpness is proved in [17] (for general martingales see [1] and [2]).

\footnotetext{
${ }^{0}$ Keywords: Bellman, dyadic maximal function, integral inequality

0 E-mail addresses: tdelis@math.uoa.gr, lefteris@math.uoc.gr

${ }^{0}$ MSC Number: 42B25
} 
One way to study inequalities satisfied by maximal operators is by using the so called Bellman function technique. This approach was first introduced in the work of Nazarov and Treil, [6], where the authors defined the function

$$
\begin{aligned}
& B_{p}(f, F, L)= \\
& \sup \left\{\frac{1}{|Q|} \int_{Q}\left(\mathcal{M}_{d} \phi\right)^{p}: \frac{1}{|Q|} \int_{Q} \phi=f, \frac{1}{|Q|} \int_{Q} \phi^{p}=F, \sup _{R: Q \subseteq R} \frac{1}{|R|} \int_{R} \phi=L\right\}
\end{aligned}
$$

with $p>1$ (as an example they examine the case $p=2$ ), where $Q$ is a fixed dyadic cube and $\phi$ is non negative in $L^{p}(Q), R$ runs over all dyadic cubes containing $Q$ and the variables $F, f, L$ satisfy $0 \leq f \leq L, f^{p} \leq F$. Exploiting a certain "pseudoconcavity" inequality it satisfies, they construct the function $4 F-4 f L+2 L^{2}$ which has the same properties as (1.5) and provides a good $L^{p}$ bound for the operator $\mathcal{M}_{d}$ (see [6] for details).

The exact value of the above Bellman function was explicitly computed for the first time by Melas in [4. In fact this was done in the much more general setting of a non-atomic probability space $(X, \mu)$ equipped with a tree structure $\mathcal{T}$, which is similar to the structure of the dyadic subcubes of $[0,1]^{n}$ (see the definition in Section (2). Then, the associated maximal operator is defined by

$$
\mathcal{M}_{\mathcal{T}} \phi(x)=\sup \left\{\frac{1}{\mu(I)} \int_{I}|\phi| \mathrm{d} \mu: x \in I \in \mathcal{T}\right\},
$$

for every $\phi \in L^{1}(X, \mu)$. Moreover (1.2) and (1.3) still hold in this setting and remain sharp.

The Bellman function of two variables related to the above maximal operator is given by

$$
B_{\mathcal{T}}^{(p)}(f, F)=\sup \left\{\int_{X}\left(\mathcal{M}_{\mathcal{T}} \phi\right)^{p} \mathrm{~d} \mu: \phi \geq 0, \int_{X} \phi \mathrm{d} \mu=f, \int_{X} \phi^{p} \mathrm{~d} \mu=F\right\},
$$

where $0<f^{p} \leq F$. In $[3$ it is proved that

$$
B_{\mathcal{T}}^{(p)}(f, F)=F \omega_{p}\left(\frac{f^{p}}{F}\right)^{p},
$$

where $\omega_{p}:[0,1] \rightarrow\left[1, \frac{p}{p-1}\right]$, is defined by $\omega_{p}(z)=H_{p}^{-1}(z)$, and $H_{p}(z)$ is given by $H_{p}(z)=-(p-1) z^{p}+p z^{p-1}$. As a consequence $B_{\mathcal{T}}^{(p)}(f, F)$ does not depend on the tree $\mathcal{T}$. The technique for the evaluation of (1.6), that is used in [4, is based on an effective linearization of the dyadic maximal operator that holds on an adequate class of functions, which is enough to describe the problem as is settled on (1.6). Using this result on suitable subsets of $X$ and several calculus arguments, the author also managed to precisely evaluate the corresponding to 
(1.4) Bellman function in this context,

$$
\begin{array}{r}
B_{\mathcal{T}}^{p}(f, F, L)=\sup \left\{\int _ { X } \left(\max \left(\mathcal{M}_{\mathcal{T}} \phi, L\right)^{p} \mathrm{~d} \mu: \phi \geq 0, \phi \in L^{p}(X, \mu),\right.\right. \\
\left.\int_{X} \phi \mathrm{d} \mu=f, \int_{X} \phi^{p} \mathrm{~d} \mu=F,\right\} .
\end{array}
$$

We wish to refine (1.3) even further. So we consider the $q$-norm, $1<q<p$, of the function $\phi$ as fixed and aim to compute the corresponding Bellman function:

$$
\begin{aligned}
& B_{\mathcal{T}}^{p, q}(f, A, F)=\sup \left\{\int_{X}\left(\mathcal{M}_{\mathcal{T}} \phi\right)^{p} \mathrm{~d} \mu: \phi \geq 0, \phi \in L^{p}(X, \mu),\right. \\
&\left.\int_{X} \phi \mathrm{d} \mu=f, \int_{X} \phi^{q} \mathrm{~d} \mu=A, \int_{X} \phi^{p} \mathrm{~d} \mu=F,\right\},
\end{aligned}
$$

where $1<q<p$, and for $f, A, F$ we have $f^{q}<A<F^{\frac{q}{p}}$. The new integral variable makes the problem considerably more difficult and thus makes it interesting to compute this Bellman function in a sub-domain of its original domain. We state our result in Theorem 1 below. The proof is an application of techniques presented in [4. So, with $\omega_{p}$ as defined above (and $\omega_{q}$ in an analogous way), in Section 3 we prove the following.

Theorem 1. For $f, A$ such that $0<f^{q}<A$ and $F=F(f, A)$ satisfying $\omega_{p}\left(\frac{f^{p}}{F}\right)=\omega_{q}\left(\frac{f^{q}}{A}\right)$, we have that

$$
B_{\mathcal{T}}^{p, q}(f, A, F)=\omega_{q}\left(\frac{f^{q}}{A}\right)^{p} F
$$

Regarding the general case now, in Section 4 we provide an upper bound for (1.9) (Lemma 4.2). As a first step towards this, we prove an inequality satisfied by the corresponding maximal operator (Lemma 4.1) which can be interpreted as the basic inequality proved in $[3$, for the case $\beta=0$.

We remark here that there are several problems in Harmonic Analysis were Bellman functions arise. Such problems (including the dyadic Carleson imbedding theorem and weighted inequalities) are described in [8] (see also [6], [7]) and also connections to Stochastic Optimal Control are provided, from which it follows that the corresponding Bellman functions satisfy certain nonlinear secondorder PDEs. The exact evaluation of a Bellman function is a difficult task which is connected with the deeper structure of the corresponding Harmonic Analysis problem. Until now several Bellman functions have been computed (see [1], 4], [5] 6], [13, 14, 15]). The exact computation of (1.6) has also been given in [12 by L. Slavin, A. Stokolos and V. Vasyunin, which linked the computation of it to solving certain PDEs of the Monge-Ampère type, and in this way they obtained an alternative proof of the results in [4 for the Bellman function related to the dyadic maximal operator. Also in [16, using the Monge- Ampère equation approach, a more general Bellman function than the one related to the 
Carleson imbedding theorem has been precisely evaluated thus generalizing the corresponding result in [4. It would be an interesting problem to discover if the Bellman function of three variables defined in (1.9) can be computed using such PDE-based methods.

\section{Preliminaries}

In this section we present the background we need from [4, that will be used in all that follows.

Let $(X, \mu)$ be a non-atomic probability space. Two measurable subsets $\mathrm{A}$, B of $X$ will be called almost disjoint if $\mu(A \cap B)=0$.

Definition 2.1. A set $\mathcal{T}$ of measurable subsets of $X$ will be called a tree if the following conditions are satisfied:

(i) $X \in \mathcal{T}$ and for every $I \in \mathcal{T}$ we have $\mu(I)>0$.

(ii) For every $I \in \mathcal{T}$ there corresponds a finite or countable subset $\mathcal{C}(I) \subseteq \mathcal{T}$ containing at least two elements such that:

(a) the elements of $\mathcal{C}(I)$ are pairwise almost disjoint subsets of $I$,

(b) $I=\bigcup \mathcal{C}(I)$.

(iii) $\mathcal{T}=\bigcup_{m \geq 0} \mathcal{T}_{m}$ where $\mathcal{T}_{(0)}=\{X\}$ and $\mathcal{T}_{(m+1)}=\bigcup_{I \in \mathcal{T}_{(m)}} \mathcal{C}(I)$.

(iv) We have $\lim _{m \rightarrow \infty} \sup _{I \in \mathcal{T}_{(m)}} \mu(I)=0$.

By induction it can be seen that each family $\mathcal{T}_{(m)}$ consists of pairwise almost disjoint sets whose union is $X$. Moreover if $x \in X \backslash E(\mathcal{T})$ then for each $m$ there exists exactly one $I_{m}(x)$ in $\mathcal{T}_{(m)}$ containing x. For every $m>0$ there is a $J \in \mathcal{T}_{(m-1)}$ such that $I_{m}(x) \in \mathcal{C}(J)$. Then, since $x \in J$, we must have $J=I_{m-1}(x)$, because $x$ does not belong to $E(\mathcal{T})$. Hence the set $\mathcal{A}=\{I \in \mathcal{T}: x \in I\}$ forms a chain $I_{0}(x)=X \supseteq I_{1}(x) \nsupseteq \ldots$ with $I_{m} \in \mathcal{C}\left(I_{m-1}(x)\right)$ for every $m>0$. From this remark it follows that if $I, J \in \mathcal{T}$ and $I \cap J \cap(X \backslash E(\mathcal{T}))$ is non-empty, then $I \subseteq J$ or $J \subseteq I$. In particular for any $I, J \in \mathcal{T}$, either $\mu(I \cap J)=0$ or one of them is contained in the other.

We will also need the following.

Lemma 2.1. For every $I \in \mathcal{T}$ and every a such that $0<a<1$ there exists a subfamily $\mathcal{F}(I) \subseteq \mathcal{T}$ consisting of pairwise almost disjoint subsets of I such that

$$
\mu\left(\bigcup_{J \in \mathcal{F}(I)} J\right)=\sum_{J \in \mathcal{F}(I)} \mu(J)=(1-a) \mu(I) .
$$

The last thing we will use is the following Lemma. Let $\omega_{q}$ be as defined in the Introduction (note that the subscript is q instead of $\mathrm{p}$ ). 
Lemma 2.2. Let $q>1$ and $\tau \in(0,1]$ be fixed. Then for every $\alpha$ with $0<\alpha<1$ the equation

$$
-(z-\alpha)^{q}+(1-\alpha)^{q-1} z^{q}=\tau \alpha(1-\alpha)^{q-1}
$$

has a unique solution $z=z(\alpha, \tau) \in[1, \infty)$ and moreover

$$
\lim _{\alpha \rightarrow 0^{+}} z(\alpha, \tau)=\omega_{q}(\tau)
$$

\section{Computation of the Bellman function on a sub-domain}

Following [4, we choose $\alpha$ with $0<\alpha<1$ and using Lemma 2.1, for every $I \in \mathcal{T}$ we choose a family $\mathcal{F}(I) \subseteq \mathcal{T}$ of pairwise almost disjoint subsets of $I$ such that

$$
\mu\left(\bigcup_{J \in \mathcal{F}(I)} J\right)=\sum_{J \in \mathcal{F}(I)} \mu(J)=(1-a) \mu(I) .
$$

Then we define $\mathcal{S}=\mathcal{S}_{\alpha}$ to be the smallest subset of $\mathcal{T}$ such that $X \in \mathcal{S}$ and for every $I \in \mathcal{S}, \mathcal{F}(I) \subseteq \mathcal{S}$ and the correspondence $I \rightarrow I^{*}$ with respect to this $\mathcal{S}$, by setting $J^{*}=I \in \mathcal{S}$ if and only if $J \in \mathcal{F}(I)$ and so writing

$$
A_{I}=I \backslash \bigcup_{J \in \mathcal{S}: J^{*}=I} J,
$$

we have $a_{I}=\mu\left(A_{I}\right)=\mu(I)-\sum_{J \in \mathcal{S}: J^{*}=I} \mu(J)=\alpha \mu(I)$ for every $I \in \mathcal{S}$. Also it is easy to see that

$$
\mathcal{S}=\bigcup_{m \geq 0} \mathcal{S}_{(m)}, \text { where } \mathcal{S}_{(0)}=\{X\} \text { and } \mathcal{S}_{(m+1)}=\bigcup_{I \in \mathcal{S}_{(m)}} \mathcal{F}(I) .
$$

The rank $r(I)$ of any $I \in \mathcal{S}$ is now defined to be the unique integer $m$ such that $I \in \mathcal{S}_{(m)}$.

For $\alpha \in(0,1)$ and the family $\mathcal{S}=\mathcal{S}_{\alpha}$ defined as above, we set

$$
x_{I}=\lambda \gamma^{r(I)} \mu(I)^{1 / q}
$$

where $\lambda=f \alpha^{-1 / \dot{q}}(1-\gamma(1-\alpha)), 1 / q+1 / \dot{q}=1, \gamma=\frac{\beta+1}{\beta+1-\beta \alpha}, \beta>0$. For every $I \in \mathcal{S}$ and every $m \geq 0$ we write

$$
b_{m}(I)=\sum_{\substack{\mathcal{S} \ni J \subseteq I \\ r(J)=r(I)+m}} \mu(J)
$$

and observing that

$$
b_{m+1}(I)=\sum_{\substack{\mathcal{S} \ni J \subseteq I, r(J)=r(I)+m}} \sum_{L \in \mathcal{F}(J)} \mu(L)=(1-\alpha) b_{m}(I)
$$


we get

$$
b_{m}(I)=(1-\alpha)^{m} \mu(I) .
$$

Hence

$$
\begin{aligned}
\sum_{I \in \mathcal{S}_{\alpha}} x_{I}^{q} & =\lambda^{q} \sum_{m \geq 0} \sum_{I \in \mathcal{S}_{(m)}} \gamma^{m q} \mu(I)=\lambda^{q} \sum_{m \geq 0} \gamma^{m q} b_{m}(X) \\
& =\lambda^{q} \sum_{m \geq 0}\left[\gamma^{q}(1-\alpha)\right]^{m}=\frac{\lambda^{q}}{1-\gamma^{q}(1-\alpha)}
\end{aligned}
$$

assuming that $\gamma^{q}(1-\alpha)<1$. Additionally,

$$
\begin{aligned}
a_{I}^{1 / q} y_{I} & =\frac{1}{\mu(I)} \sum_{\substack{J \in \mathcal{S} \\
J \subseteq I}} a_{I}^{1 / q} a_{J}^{1 / \hat{q}} x_{J} \\
& =\frac{\lambda}{\mu(I)} \sum_{\substack{J \in \mathcal{S} \\
J \subseteq I}}(\alpha \mu(I))^{1 / q}(\alpha \mu(J))^{1 / \dot{q}} \gamma^{r(J)} \mu(J)^{1 / q} \\
& =\frac{\alpha \lambda}{\mu(I)^{1-\frac{1}{q}}} \sum_{m \geq 0} \gamma^{m+r(I)} \sum_{\substack{\mathcal{S} \ni J \subseteq I \\
r(J)=r(I)+m}} \mu(J) \\
& =\frac{\alpha \lambda}{\mu(I)^{1-\frac{1}{q}}} \gamma^{r(I)} \sum_{m \geq 0} \gamma^{m} \mu(I)(1-\alpha)^{m}=\frac{\alpha}{(1-\gamma(1-\alpha))} x_{I},
\end{aligned}
$$

where the $y_{I}$ 's are defined by the first equality above.

Now we consider the function

$$
\varphi_{\alpha}=\sum_{I \in \mathcal{S}} \frac{x_{I}}{a_{I}^{1 / q}} \chi_{A_{I}}
$$

$I \in \mathcal{S}$. It is easy to see that

$$
\int_{X} \phi_{\alpha} \mathrm{d} \mu=f
$$

and writing $z=\beta+1-\beta \alpha$ and thus $\gamma=\frac{\beta+1}{\beta+1-\beta \alpha}=\frac{z-\alpha}{z(1-\alpha)}$,

$$
\int_{X} \phi_{\alpha}^{q} \mathrm{~d} \mu=\sum_{I \in \mathcal{S}} x_{I}^{q}=\frac{f^{q} \alpha(1-\alpha)^{q-1}}{-(z-\alpha)^{q}+(1-\alpha)^{q-1} z^{q}} .
$$

We are now in a position to prove Theorem 11. Let $\phi_{\alpha}$ be as in (3.10) and $z=z\left(\alpha, \frac{f^{q}}{A}\right)$ the solution provided by Lemma 2.2 which makes $\int_{X} \phi_{\alpha}^{q} \mathrm{~d} \mu=A$ (and also $\left.\gamma^{q}(1-\alpha)=\frac{(z-\alpha)^{q}}{z^{q}(1-\alpha)^{q-1}}=1-\frac{\alpha f^{q}}{z^{q} A}<1\right)$. Set $F(\alpha)=\int_{X} \phi_{\alpha}^{p} \mathrm{~d} \mu=$ $\sum_{I \in \mathcal{S}_{\alpha}} a_{I}^{-\frac{p-q}{q}} x_{I}^{p}$. Since

$$
\mathcal{M}_{\mathcal{T}} \phi_{\alpha} \geq \sum_{I \in \mathcal{S}}\left(\frac{1}{\mu(I)} \int_{I} \phi_{\alpha} \mathrm{d} \mu\right) \chi_{A_{I}}=\sum_{I \in \mathcal{S}} \frac{1}{\mu(I)}\left(\sum_{\substack{J \in \mathcal{S} \\ J \subseteq I}} a_{J}^{1 / \dot{q}} x_{J}\right) \chi_{A_{I}}
$$


from (3.9) we have

$$
\int_{X}\left(\mathcal{M}_{\mathcal{T}} \phi_{\alpha}\right)^{p} \mathrm{~d} \mu \geq \sum_{I \in \mathcal{S}} a_{I} y_{I}^{p}=z^{p} F(\alpha)
$$

From Lemma 2.2, $z \rightarrow \omega_{q}\left(\frac{f^{q}}{A}\right)$ and, arguing as in (3.8),

$$
F(\alpha)=\frac{f^{p} \alpha(1-\alpha)^{p-1}}{-(z-\alpha)^{p}+(1-\alpha)^{p-1} z^{p}} \rightarrow \frac{f^{p}}{H_{p}\left(\omega_{q}\left(\frac{f^{q}}{A}\right)\right)}=F(f, A)
$$

as $\alpha \rightarrow 0^{+}$. So taking limits in (3.13), we conclude that

$$
B_{\mathcal{T}}^{p, q}(f, A, F(f, A)) \geq \omega_{q}\left(\frac{f^{q}}{A}\right)^{p} F(f, A) .
$$

To see that the converse inequality also holds, we use (1.7), our hypothesis on the relation between $f, A, F$, and the inequality

$$
B_{\mathcal{T}}^{p, q}(f, A, F) \leq B_{\mathcal{T}}^{(p)}(f, F)
$$

which holds by the corresponding definitions. So finally,

$$
B_{\mathcal{T}}^{p, q}(f, A, F(f, A))=\omega_{q}\left(\frac{f^{q}}{A}\right)^{p} F(f, A)
$$

which is what we aimed to prove.

\section{Upper bound for (1.9)}

We first prove an inequality (Lemma 4.1) satisfied by the maximal operator defined in (1.5). For this proof we use a variation of the approach that arises in 9], as can be seen below. An application of this, in case one also considers the $L^{p}$-norm of $\phi$ as given, provides us with an upper bound to (1.9). This is the content of Lemma 4.2

Lemma 4.1. If $I=\int_{X}\left(\mathcal{M}_{\mathcal{T}} \phi\right)^{p} \mathrm{~d} \mu$ and $\int_{X} \phi \mathrm{d} \mu=f, \int_{X} \phi^{q} \mathrm{~d} \mu=A$ then,

$$
I \leq f^{p}-\frac{p}{p-q} f^{p-q} A+\frac{p}{p-q} \int_{X}\left(\mathcal{M}_{\mathcal{T}} \phi\right)^{p-q} \phi^{q} \mathrm{~d} \mu .
$$

Proof. We have

$$
\begin{aligned}
I & =\int_{\lambda=0}^{\infty} p \lambda^{p-1} \mu\left(\left\{\mathcal{M}_{\mathcal{T}} \phi \geq \lambda\right\}\right) \mathrm{d} \lambda \\
& =\int_{\lambda=0}^{f} p \lambda^{p-1} \mu\left(\left\{\mathcal{M}_{\mathcal{T}} \phi \geq \lambda\right\}\right) \mathrm{d} \lambda+\int_{\lambda=f}^{\infty} p \lambda^{p-1} \mu\left(\left\{\mathcal{M}_{\mathcal{T}} \phi \geq \lambda\right\}\right) \mathrm{d} \lambda \\
& =I_{1}+I_{2} .
\end{aligned}
$$


Then, since $\left\{\mathcal{M}_{\mathcal{T}} \phi \geq \lambda\right\}=X$, for every $\lambda<f$ (because $\mathcal{M}_{\mathcal{T}} \phi(x) \geq f$, for every $x \in X)$, we have

$$
I_{1}=\int_{\lambda=0}^{f} p \lambda^{p-1} \mathrm{~d} \lambda=f^{p}
$$

and

$$
I_{2}=\int_{\lambda=f}^{\infty} p \lambda^{p-1} \mu\left(\left\{\mathcal{M}_{\mathcal{T}} \phi \geq \lambda\right\}\right) \mathrm{d} \lambda
$$

For $\lambda>f$ set $E_{\lambda}=\left\{\mathcal{M}_{\mathcal{T}} \phi \geq \lambda\right\}=\cup I_{j}$, where $I_{j} \in \mathcal{T}$ maximal with respect to the condition $\frac{1}{\mu\left(I_{j}\right)} \int_{I_{j}} \phi \mathrm{d} \mu \geq \lambda$. Since by maximality the $I_{j}$ 's are pairwise disjoint, $\mu\left(E_{\lambda}\right)=\sum \mu\left(I_{j}\right) \leq \sum_{j} \frac{1}{\lambda} \int_{I_{j}} \phi \mathrm{d} \mu=\frac{1}{\lambda} \int_{\cup I_{j}} \phi \mathrm{d} \mu=\frac{1}{\lambda} \int_{E_{\lambda}} \phi \mathrm{d} \mu$. So

$$
\mu\left(E_{\lambda}\right) \leq \frac{1}{\lambda} \int_{E_{\lambda}} \phi \mathrm{d} \mu
$$

which is the general weak-type $(1,1)$ inequality for the dyadic maximal operator. From (4.4) we conclude that $\frac{1}{\mu\left(E_{\lambda}\right)} \int_{E_{\lambda}} \phi \mathrm{d} \mu \geq \lambda$ and consequently, for $1<q<p$, by using Hölder's inequality, we obtain $\lambda^{q} \leq\left(\frac{1}{\mu\left(E_{\lambda}\right)} \int_{E_{\lambda}} \phi \mathrm{d} \mu\right)^{q} \leq$ $\frac{1}{\mu\left(E_{\lambda}\right)} \int_{E_{\lambda}} \phi^{q} \mathrm{~d} \mu$ and so

$$
\mu\left(E_{\lambda}\right) \leq \frac{1}{\lambda^{q}} \int_{E_{\lambda}} \phi^{q} \mathrm{~d} \mu .
$$

Inserting (4.5) in (4.3), we get

$$
\begin{aligned}
I_{2} & \leq \int_{\lambda=f}^{\infty} p \lambda^{p-1} \frac{1}{\lambda^{q}} \int_{\left\{\mathcal{M}_{\mathcal{T} \phi \geq \lambda\}}\right.} \phi^{q} \mathrm{~d} \mu \mathrm{d} \lambda \\
& =\int_{X} p \phi(x)^{q}\left(\int_{\lambda=f}^{\mathcal{M}_{\mathcal{T} \phi(x)}} \lambda^{p-q-1} \mathrm{~d} \lambda\right) \mathrm{d} \mu(x)= \\
& =\frac{p}{p-q} \int_{X} \phi(x)^{q}\left[\lambda^{p-q}\right]_{\lambda=f}^{\mathcal{M}_{\mathcal{T}} \phi(x)} \mathrm{d} \mu(x) \\
& =\frac{p}{p-q} \int_{X}\left(\mathcal{M}_{\mathcal{T}} \phi\right)^{p-q} \phi^{q} \mathrm{~d} \mu-\frac{p}{p-q} f^{p-q} \int_{X} \phi^{q} \mathrm{~d} \mu \\
& =\frac{p}{p-q} \int_{X}\left(\mathcal{M}_{\mathcal{T}} \phi\right)^{p-q} \phi^{q} \mathrm{~d} \mu-\frac{p}{p-q} f^{p-q} A .
\end{aligned}
$$

From (4.6) and (4.1), (4.2) we finally get

$$
I=\int_{X}\left(\mathcal{M}_{\mathcal{T}} \phi\right)^{p} \mathrm{~d} \mu \leq f^{p}-\frac{p}{p-q} f^{p-q} A+\frac{p}{p-q} \int_{X}\left(\mathcal{M}_{\mathcal{T}} \phi\right)^{p-q} \phi^{q} \mathrm{~d} \mu
$$

which is the desired inequality.

Lemma 4.2. If $\int_{X} \phi \mathrm{d} \mu=f, \int_{X} \phi^{q} \mathrm{~d} \mu=A$ and $\int_{X} \phi^{p} \mathrm{~d} \mu=F$, then

$$
I=\int_{X}\left(\mathcal{M}_{\mathcal{T}} \phi\right)^{p} \mathrm{~d} \mu \leq F h^{-1}\left(\frac{p f^{p-q} A-(p-q) f^{p}}{F}\right)^{p},
$$

where $h:[1, \infty) \rightarrow(-\infty, q]$ with $h(t)=p t^{p-q}-(p-q) t^{p}$. 
Proof. From (4.7), after using Hölder inequality for the integral on the right hand side, we have

$$
I=\int_{X}\left(\mathcal{M}_{\mathcal{T}} \phi\right)^{p} \mathrm{~d} \mu \leq f^{p}-\frac{p}{p-q} f^{p-q} A+\frac{p}{p-q} I^{\frac{p-q}{p}} F^{\frac{q}{p}},
$$

which, if we divide by $\mathrm{F}$ becomes

$$
(p-q) \frac{I}{F} \leq \frac{(p-q) f^{p}-p f^{p-q} A}{F}+p\left(\frac{I}{F}\right)^{\frac{p-q}{p}}
$$

and this gives

$$
p\left(\frac{I}{F}\right)^{\frac{p-q}{p}}-(p-q)\left(\frac{I}{F}\right) \geq k(f, A, F),
$$

where $k(f, A, F)=\left(p f^{p-q} A-(p-q) f^{p}\right) / F$. So

$$
\begin{gathered}
p\left[\left(\frac{I}{F}\right)^{\frac{1}{p}}\right]^{p-q}-(p-q)\left[\left(\frac{I}{F}\right)^{\frac{1}{p}}\right]^{p} \geq k(f, A, F) \Rightarrow \\
h\left[\left(\frac{I}{F}\right)^{\frac{1}{p}}\right] \geq k(f, A, F) .
\end{gathered}
$$

We consider now the function $h(t)=p t^{p-q}-(p-q) t^{p}, t>0$. We observe that

$$
h^{\prime}(t)=p(p-q) t^{p-q-1}-p(p-q) t^{p-1}=p(p-q) t^{p-q-1}\left(1-t^{q}\right) .
$$

So $h$ is strictly decreasing on $[1, \infty)$, and strictly increasing on $(0,1]$. Additionally, $h(1)=q$ while $\lim _{t \rightarrow \infty} h(t)=-\infty$. Thus $h(t) \leq q$, for any $t>0$. At the same time

$$
\begin{aligned}
k(f, A, F) & =\frac{p f^{p-q} A-(p-q) f^{p}}{F}=\frac{-(p-q)\left(\frac{f}{A^{1 / q}}\right)^{p}+p\left(\frac{f}{A^{1 / q}}\right)^{p-q}}{F} A^{\frac{p}{q}} \\
& =\frac{-(p-q) \tau^{p}+p \tau^{p-q}}{F} A^{\frac{p}{q}}=\left[\frac{h(\tau)}{F}\right] A^{\frac{p}{q}} \leq q \frac{A^{\frac{p}{q}}}{F} \leq q,
\end{aligned}
$$

where $\tau=\frac{f}{A^{1 / q}}$. So

$$
q \geq k(f, A, F)>0
$$

and (4.9) yields

$$
\left(\frac{I}{F}\right)^{\frac{1}{p}} \leq h^{-1}(k(f, A, F))
$$

which is what we wanted.

\section{References}

[1] D. L. Burkholder, Martingales and Fourier Analysis in Banach spaces, C.I.M.E. Lectures, Varenna, Como, Italy, 1985, Lecture Notes Math. 1206 (1986), 81-108. 
[2] D. L. Burkholder, Explorations in martingale theory and its applications, École d' Été de Probabilitiés de Saint-Flour XIX-1989, Lecture Notes Math. 1464 (1991), 1-66.

[3] A. D. Delis, E. N. Nikolidakis Sharp integral inequalities for the dyadic maximal operator and applications, submitted, arXiv: 1502.06002v7

[4] A. D. Melas, The Bellman functions of dyadic-like maximal operators and related inequalities, Adv. in Math. 192 (2005), 310-340.

[5] A. D. Melas, Sharp general local estimates for dyadic-like maximal operators and related Bellman functions, Adv. in Math. 220 (2009),No 2 367-426.

[6] F. Nazarov, S. Treil, The hunt for a Bellman function: Applications to estimates for singular integral operators and to other classical problems of harmonic analysis, St. Petersburg Math. J. 8 no. 5 (1997), 721-824

[7] F. Nazarov, S. Treil and A. Volberg, The Bellman functions and two-weight inequalities for Haar multipliers, Journ. Amer. Math. Soc. 12 no. 4 (1999), 909-928.

[8] F. Nazarov, S. Treil and A. Volberg, Bellman function in stochastic optimal control and harmonic analysis (how our Bellman function got its name), Oper. Theory: Advances and Appl. 129 (2001), 393-424, Birkhauser, Verlag. MR1882704 (2003b:49024).

[9] E. N. Nikolidakis, A. D. Melas, A sharp integral rearrangement inequality for the dyadic maximal operator and applications, Appl. and Comp. Harmonic Anal., 38 (2015), Issue 2, 242-261.

[10] E. Nikolidakis, Optimal weak type estimates for dyadic-like maximal operators, Ann. Acad. Scient. Fenn. Math. 38 (2013), 229-244.

[11] E. Nikolidakis, Sharp weak type inequalities for the dyadic maximal operator, J. Fourier. Anal. Appl., 19 (2012), 115-139.

[12] L. Slavin, A. Stokolos, V. Vasyunin, Monge-Ampère equations and Bellman functions: The dyadic maximal operator C. R. Math. Acad. Sci. Paris Sér. I. 346 (2008), 585-588.

[13] L. Slavin, A. Volberg, The explicit BF for a dyadic Chang-Wilson-Wolff theorem. The s-function and the exponential integral, Contemp. Math. 444. Amer. Math. Soc., Providence, RI, 2007.

[14] V. Vasyunin, The sharp constant in the reverse Hölder inequality for Muckenhoupt weights, St. Petersburg Math. J., 15 (2004), no. 1, 49-75.

[15] V. Vasyunin, A. Volberg, The Bellman functions for a certain two weight inequality: The case study, St. Petersburg Math. J., 18 (2007), No. 2, p 201-222. 
[16] V. Vasyunin, A. Volberg, Monge-Ampère equation and Bellman optimization of Carleson embedding theorems, Linear and complex analysis, 195238, Amer. Math. Soc. Transl. Ser.2, 226, Amer. Math. Soc., Providence, RI, 2009.

[17] G. Wang, Sharp maximal inequalities for conditionally symmetric martingales and Brownian motion, Proc. Amer. Math. Soc. 112 (1991), 579-586.

Anastasios D. Delis, Eleftherios N. Nikolidakis, National and Kapodistrian University of Athens, Department of Mathematics, Panepistimioupolis, Zografou 157 84, Athens, Greece. 